Bangladesh J. Pl. Breed. Genet., 27(1): 01-08, 2014

\title{
COMBINING ABILITY AND HETEROSIS IN QUASI AROMATIC RICE (Oryza sativa L.)
}

\author{
M. Ali, M. A. K. Mian, M. A. Hossain ${ }^{1}$, M. Z. Islam $^{1}$ and M. Manzur Hossain ${ }^{2}$ \\ Department of Genetics and Plant Breeding \\ Bangabandhu Sheikh Mujibur Rahman Agricultural University \\ Gazipur 1706, Bangladesh
}

\begin{abstract}
The experiment was conducted to study general and specific combining ability (GCA and SCA) and to estimate heterosis estimation at experimental farm of Bangabandhu Sheikh Mujibur Rahman Agricultural University, Gazipur during T. Aman, 2006 and 2007 seasons. Eleven rice parental lines consisting of two quasi CMS lines in $\mathrm{BC}_{3}$ generation as lines and nine restorer lines as testers along with their eighteen $\mathrm{F}_{1} \mathrm{~s}$ and a check variety (BRRI dhan37) were used for combining ability study in a line $\mathrm{x}$ tester mating design for eight characters. Ratio of general combining ability (GCA) to specific combining ability (SCA) variances above unity were observed for 1000-grain weight $(\mathrm{g})$ indicating predominance of additive gene action for controlling the character. Among mother parents, CMS-1 appeared as good general combiner for panicle length, 1000-grain weight $(\mathrm{g})$, and grain length-breadth ratio. Among male parents, Benaful, Oval Tapi and Sorukamini-1 were observed to be good general combiners for most of the characters studied. The cross combinations, CMS $-1 \times$ Kataribhog, CMS- $2 \times$ Maloti- 1 and CMS- $2 \times$ Keora were found as good specific combiners for most of the characters under study. Good specific combiners were evolved from all kinds of combinations of general combiners indicating additive and non-additive types of gene effect for inheritance. Maximum hybrids showed desired significant standard heterosis for panicle length and 1000-grain weight. None of the hybrids showed desired and significant standard heterosis simultaneously for any three or more characters.
\end{abstract}

Key words: GCA; SCA; heterosis; CMS lines; aromatic rice

\section{INTRODUCTION}

Application of heterosis breeding in rice was limited because of self-pollination character of the plant until 1980 decades. Heterosis in rice came to practical use since discovery of cytoplasmic-genetic male sterility (CMS) system (Yuan 1998). Now, this technology is also important for aromatic rice growing countries to increase the productivity of aromatic rice. Estimation of different types of heterosis is helpful for the breeder to observe superiority of the hybrids in respect of their parents or standard varieties. The extent of standard heterosis is one of the critical factors that decide the economic viability of a hybrid technology. High heterobeltiosis or high standard heterosis in panicle and grain traits is prerequisite for a good hybrid. It also needs to reduce spikelet sterility and increase grain weight with higher heterosis for number of

${ }^{1}$ Plant Breeding Division, Bangladesh Rice Research Institute, Gazipur 1701

${ }^{2}$ Agriculture Information Service, Khamarbari, Farmgate, Dhaka 1215 
spikelets per panicle in hybrid rice (Sitaramaiah et al., 1998). Evaluation of promising Cytoplasmic male sterile (CMS) and restorer lines for their combining ability is essential to identify most promising yield contributing hybrid. Based on combining ability, different researchers (Mehla et al., 2000; Sarker et al., 2002; Swamy et al., 2003) found different values of GCA and SCA effects in parents and hybrids for different characters. Such information helps to choose good parents as well as combinations. Therefore, the experiment was undertaken to assess the nature and extent of combining ability of selected parents and their hybrids and to estimate the magnitude and direction of heterosis in aromatic hybrid rice.

\section{MATERIALS AND METHODS}

The experiment was carried out at Bangabandhu Sheikh Mujibur Rahman Agricultural University, Gazipur during T. Aman 2006 and 2007. In T. Aman, 2006 season two quasi CMS lines, CMS-1 (IR62829A $\times$ Elai in $\mathrm{BC}_{3}$ ) and CMS-2 (IR62829A $\times$ Kalijira-2 in $\mathrm{BC}_{3}$ ) were grown separately each surrounded by one restorer in a plot. Nine restorers viz; Benaful, Chinigura, Kalijira-4, Kalizira-7, Kahataribhog, Keora, Maloti-1, Oval Tapi and Sorukamini-1 were used in nine plots for every CMS line. So, there were eighteen plots. Restorers were ten days older than CMS lines as to synchronize flowering for natural crossing. Each plot was surrounded by white cloths as a barrier for unwanted pollination. At heading stage, pollen sterility of every plant of each CMS lines in every plot was tested to confirm their male sterility status. Plants with fertile pollen at any percentage were rejected. Mature seeds from CMS parents were collected as hybrid seeds and stored in proper storage. Thus, eleven parents consisting two quasi CMS lines and nine restores with their hybrids and a check variety BRRI dhan-37 were grown in T Aman, 2007 season. The experiment was conducted in randomized complete block design (RCBD) with three replications was followed for this investigation. Data were collected from 10 randomly selected hills per plot on plant height $(\mathrm{cm})$, number of productive tillers per plant, panicle length $(\mathrm{cm})$, number of total spikelets per panicle, spikelet fertility, number of filled grains per panicle, 1000-grain weight $(\mathrm{g})$, yield per hill $(\mathrm{g})$ and grain length-breadth ratio. Days to maturity were recorded from each of the plots. Combining ability analysis was calculated using line $\times$ tester method suggested by Kempthorne (1957). Significance of GCA and SCA was tested by ' $\mathrm{t}$ ' test. Standard heterosis was estimated following the formula according to Fehr (1987).

\section{RESULTS AND DISCUSSION}

Variance due to parents vs. crosses was significant for all the characters suggesting the presence of significant heterotic effect. Mean sum of squares resulted for line, tester and line $\times$ tester were also significant for all the characters which revealed wide range of variability (Table 1) except plant height. GCA variances were found significant for spikelet fertility, 1000-grain weight (g), yield (g/plant) and grain lengthbreadth ratio while SCA variances were found significant for all characters except plant height. It indicated that both additive and non-additive types of gene action for spikelet fertility index, 1000 grain weight and grain length-breadth ratio while non-additive type of gene action for panicle length, number of total spikelets per panicle and number of filled grains per panicle. GCA/SCA above unity was observed for 1000-grain weight indicating predominance of additive gene action for controlling this character while ratio of GCA to SCA variance below unity was estimated for other characters which revealed predominance of non-additive gene effect in inheritance of those characters. Ratio of 
GCA to SCA variance above unity was also observed by Surek and Korkut (1996) for 1000-grain weight while ratio of GCA to SCA variance below unity was reported by Bansal et al. (2000) for panicle length and Swamy et al. (2003) for number of total spikelets per panicle, number of filled grains per panicle and spikelet fertility.

Estimates of GCA effects revealed that none of the parents was good general combiner for all characters in desired direction (Table 2). Between two quasi CMS lines, CMS-1 appeared with good general combining ability for panicle length, 1000- grain weight and grain length-breadth ratio while CMS-2 showed good general combining ability for number of total spikelets per panicle and number of filled grains per panicle. Among testers, Benaful showed good general combining ability for spikelet fertility index, 1000-grain weight and grain length-breadth ratio while Oval Tapi showed the same for panicle length, 1000-grain weight $(\mathrm{g})$, yield (g/plant) and grain length-breadth ratio. Sorukamini-1 also appeared as good general combiner for number of total spikelets per panicle, number of filled grains per panicle, spikelet fertility and grain lengthbreadth ratio. Sarker et al. (2002) denoted IR58025A and IR44675R and Faiz et al. (2006) identified IR69616A and Basmati385 as good general combiners. Other testers became as good general combiners for at least two characters. GCA effect for more than one desirable character indicated their utility in heterosis breeding program. Cross combination, CMS- $1 \times$ Sorukamini- 1 was good specific combiner for seven charaters in desired direction (Table 3). Similarly, CMS- $2 \times$ Keora and CMS $-1 \times$ Kataribhog were good specific combiners for five and seven characters, respectively. These good specific combiners were evolved from good $\times$ good and poor $\times$ poor general combiners which were due to additive and non-additive gene actions. Similarly, Yadav et al. (1999) found IR62829A $\times$ IET13610 and Mehla et al. (2000) identified IR58025A $\times$ PAU1106-6-2 as best specific combiners.

On the other hand, CMS-2 $\times$ Chinigura exhibited good specific combining ability for panicle length, number of filled grains per panicle, 1000-grain weight and grain length-breadth ratio and CMS- $1 \times$ Kalijira-7 also demonstrated good specific combining ability for five characters. These specific combiners were resulted from good $\times$ good, good $\times$ average, good $\times$ poor, poor $\times$ good and poor $\times$ poor general combiners which were due to additive and non-additive gene actions. However, considering better performance, it revealed that both additive and non-additive types of gene action were involved for inheritance of different characters in hybrids. Ramalingam et al. (1997) and Sarker et al. (2002) revealed the same that superior SCA effects were produced by involving all kinds of combination of general combiners.

The nature and extent of heterosis over pollen parent and standard variety (BRRI Dhan-37) exhibited varied picture. None of the hybrids were top heterotic for all characters simultaneously. Most of the hybrids performed desired standard heterosis for all characters except number of total spikelets per panicle, number of filled grains per panicle and spikelet fertility while majority hybrids showed desired and significant heterosis over standard check variety only for panicle length and 1000-grain weight (Table 4). Maximum hybrids also showed desired and significant both types of heterosis for panicle length and 1000-grain weight. Our results agreed with the findings of Mishra and Pandey (1998) for panicle length and 1000-grain weight.

The highest standard heterosis for number of filled grains per panicle were recorded as $16.76 \%$ (CMS-2 $\times$ Maloti-1 (Table 4). Four hybrids exhibited significant standard heterosis for this character. None of them was common in both cases. Faiz et al. (2006) and Panwar et al. (1998) observed significant positive heterobeltiosis and standard heterosis, respectively for number of filled grains per panicle. 
Table 1. Analysis of variance for yield and yield contributing characters in aromatic rice under line $\mathbf{x}$ tester mating design

\begin{tabular}{|c|c|c|c|c|c|c|c|c|c|c|c|}
\hline $\begin{array}{c}\text { Sources } \\
\text { of } \\
\text { variation }\end{array}$ & df & $\begin{array}{c}\text { Plant } \\
\text { height } \\
(\mathbf{c m})\end{array}$ & $\begin{array}{c}\text { No. of } \\
\text { productive } \\
\text { tillers /plant }\end{array}$ & $\begin{array}{c}\text { Days to } \\
\text { maturity }\end{array}$ & $\begin{array}{c}\text { Panicle } \\
\text { length } \\
\text { (cm) }\end{array}$ & $\begin{array}{c}\text { No. of } \\
\text { total spikelets } \\
\text { /panicle }\end{array}$ & $\begin{array}{l}\text { Spikelet } \\
\text { fertility }\end{array}$ & $\begin{array}{l}\text { No. of filled } \\
\text { grains } \\
\text { /panicle }\end{array}$ & $\begin{array}{l}1000 \text { grain } \\
\text { weight }(\mathrm{g})\end{array}$ & $\begin{array}{c}\text { Yield } \\
\text { /plant } \\
\text { (g) }\end{array}$ & $\begin{array}{c}\text { Grain } \\
\text { length } \\
\text { breadth } \\
\text { ratio }\end{array}$ \\
\hline Replication & 2 & 4.23 & 0.07 & 0.05 & 0.08 & 14.46 & 0.00 & 2.50 & 0.04 & 0.07 & 0.00 \\
\hline Treatments & 28 & $163.91 * *$ & $5.22 * *$ & $38.25 * *$ & $16.08 * *$ & 1942.20 ** & $0.09 * *$ & $4336.04 * *$ & $37.74 * *$ & $141.38 * *$ & $0.94 * *$ \\
\hline Parents & 10 & $236.05 * *$ & $7.70 * *$ & $67.291 * *$ & 32.30 ** & $2626.11 * *$ & $0.23 * *$ & $10027.77 * *$ & $61.96 * *$ & $202.09 * *$ & 1.36 ** \\
\hline Cross & 17 & $18.24 *$ & $2.89 * *$ & $16.41 * *$ & $5.99 * *$ & $1599.52 * *$ & $0.01 * *$ & $1171.87 * *$ & $22.81 * *$ & $19.28 * *$ & $0.49 * *$ \\
\hline $\begin{array}{l}\text { Parents Vs } \\
\text { Crosses }\end{array}$ & 1 & $2463.69 * *$ & $25.61 * *$ & $149.04 * *$ & $38.66^{* *}$ & $2714.34 * *$ & $0.12 * *$ & $2219.19 * *$ & $52.26 * *$ & $1621.39 * *$ & $4.45^{* *}$ \\
\hline Line & 1 & $42.95^{*}$ & $2.76^{* *}$ & $12.52 * *$ & $12.30 * *$ & $2536.14 * *$ & $0.00 *$ & $1614.85^{* *}$ & $162.66^{* *}$ & $56.67 * *$ & $0.94 * *$ \\
\hline Tester & 8 & 17.83 & 3.12 *** & $20.57 * *$ & $4.77^{* *}$ & 1601.27 ** & $0.01 * *$ & $1376.22 * *$ & $22.36^{* *}$ & $16.67 * *$ & $0.67 * *$ \\
\hline Line $\mathrm{x}$ Tester & 8 & 15.56 & $2.67 * *$ & $12.67 * *$ & $6.42 * *$ & $1493.18^{* * *}$ & $0.00 * *$ & $912.14 * *$ & $5.77 * *$ & $17.22 * *$ & $0.25 * *$ \\
\hline Error & 56 & 9.58 & 0.10 & 0.53 & 0.23 & 31.37 & 0.00 & 1794 & 0.05 & 0.20 & 0.00 \\
\hline$\sigma^{2}$ gca & 10 & 0.90 & 0.02 & 0.23 & 0.13 & 31.85 & $0.00^{* *}$ & 35.34 & $5.26 * *$ & $1.18^{* * *}$ & $0.03^{* * *}$ \\
\hline$\sigma^{2}$ sca & 17 & 2.00 & $0.86^{* * *}$ & $4.08 * *$ & $2.06^{* *}$ & $487.27 * *$ & $0.00 * *$ & $298.07 * *$ & $1.91 * *$ & $5.67 * *$ & $0.01 * *$ \\
\hline$\sigma^{2} \mathrm{~A}$ & & 1.80 & 0.03 & 0.46 & 0.26 & $63.70 *$ & $0.00^{* *}$ & $70.72 * *$ & $10.51^{* *}$ & $2.36^{* * *}$ & $0.07 * *$ \\
\hline$\sigma^{2} D$ & & 2.00 & $0.86 * *$ & $4.08 * *$ & $2.06 * *$ & $487.27 * *$ & $0.00 * *$ & $298.07 * *$ & $1.91 * *$ & $5.67 * *$ & $0.01 * *$ \\
\hline$\sigma^{2} \mathrm{gca} / \sigma^{2} \mathrm{sca}$ & & 0.45 & 0.02 & 0.06 & 0.06 & 0.07 & 0.48 & 0.12 & 2.76 & 0.21 & 0.41 \\
\hline
\end{tabular}

Table 2. Estimate of GCA effects of parents for yield and yield contributing characters in aromatic rice

\begin{tabular}{|c|c|c|c|c|c|c|c|c|c|c|}
\hline Parent & $\begin{array}{c}\text { Plant } \\
\text { height } \\
(\mathbf{c m})\end{array}$ & $\begin{array}{c}\text { No. of } \\
\text { productive } \\
\text { tillers /plant }\end{array}$ & $\begin{array}{l}\text { Days to } \\
\text { maturity }\end{array}$ & $\begin{array}{c}\text { Panicle } \\
\text { length } \\
(\mathrm{cm})\end{array}$ & $\begin{array}{l}\text { No. of total } \\
\text { spikelets } \\
\text { /panicle } \\
\end{array}$ & $\begin{array}{l}\text { Spikelet } \\
\text { fertility }\end{array}$ & $\begin{array}{l}\text { No. of filled } \\
\text { grains } \\
\text { /panicle }\end{array}$ & $\begin{array}{c}1000 \\
\text { grain } \\
\text { weight }(g)\end{array}$ & $\begin{array}{c}\text { Yield } \\
\text { /plant (g) }\end{array}$ & $\begin{array}{c}\text { Grain length - } \\
\text { breadth ratio }\end{array}$ \\
\hline CMS-1 & -0.89 & $-0.23 * *$ & $-0.48 * *$ & $0.48 * *$ & $-6.72 * *$ & -0.01 & $-5.47 * *$ & $1.74 * *$ & $1.02 * *$ & $0.14 * *$ \\
\hline CMS-2 & 0.89 & $0.23 * *$ & $0.48 * *$ & $-0.48 * *$ & $6.72 * *$ & 0.01 & $5.47 * *$ & $-1.74 * *$ & $-1.02 * *$ & $-0.14 * *$ \\
\hline Benaful & 0.31 & 0.14 & $3.93 * *$ & $-0.69 * *$ & $-19.0524 * *$ & $0.04 * *$ & $-8.53 * *$ & $0.54 * *$ & $1.55 * *$ & $0.42 * *$ \\
\hline Chinigura & 2.41 & $1.42 * *$ & $-1.91 * *$ & $0.89 * *$ & $6.0623 *$ & $-0.03 * *$ & -0.64 & $-1.93 * *$ & $0.72 * *$ & $-0.59 * *$ \\
\hline Kalijira-4 & -1.95 & $-0.56^{* *}$ & 0.26 & 0.01 & $16.45^{* *}$ & $0.04 * *$ & $19.36^{* *}$ & $-2.25 * *$ & $1.82 * *$ & $-0.20 * *$ \\
\hline Kalijira-7 & $-2.68^{*}$ & $0.62 * *$ & $-2.07 * *$ & $1.03 * *$ & 1.73 & $-0.05 * *$ & $-7.51 * *$ & $-1.04 * *$ & $0.61 * *$ & $0.23 * *$ \\
\hline Kataribhog & -1.34 & $0.31 *$ & $0.76^{* *}$ & -0.12 & $-14.77 * *$ & $0.04 * *$ & $-5.28 * *$ & -0.03 & $-2.50 * *$ & $0.21 * *$ \\
\hline Keora & 1.03 & $-0.53 * *$ & $-1.41 * *$ & $0.54 * *$ & $-24.67 * *$ & $-0.03 * *$ & $-24.23 * *$ & $2.49 * *$ & $-2.61 * *$ & $0.14 * *$ \\
\hline Maloti-1 & 1.70 & $-0.84 * *$ & -0.24 & $-1.30 * *$ & $13.20 * *$ & $0.04 * *$ & $17.42 * *$ & $-1.21 * *$ & $-0.78 * *$ & $-0.43 * *$ \\
\hline Oval Tapl & -0.47 & $-0.58 * *$ & $1.09 * *$ & $0.82 * *$ & -0.40 & $-0.05 * *$ & $-8.86 * *$ & $3.47 * *$ & $1.41 * *$ & $0.20 * *$ \\
\hline Sorukamini-1 & 0.97 & 0.02 & -0.41 & $-1.18 * *$ & $21.46^{* *}$ & $0.01 * *$ & $18.27 * *$ & -0.02 & -0.22 & $0.02 * *$ \\
\hline
\end{tabular}

** and * significant at $1 \%$ and $5 \%$ level, respectively 
Table 3. Estimate of SCA effects of hybrids for yield and yield contributing characters in aromatic rice

\begin{tabular}{|c|c|c|c|c|c|c|c|c|c|c|}
\hline Hybrid & $\begin{array}{l}\text { Plant } \\
\text { height } \\
(\mathbf{c m})\end{array}$ & $\begin{array}{c}\text { No. of } \\
\text { productive } \\
\text { tillers/ plant }\end{array}$ & $\begin{array}{l}\text { Days to } \\
\text { maturity }\end{array}$ & $\begin{array}{l}\text { Panicle } \\
\text { length } \\
\text { (cm) }\end{array}$ & $\begin{array}{l}\text { No. of total } \\
\text { spikelets } \\
\text { /panicle }\end{array}$ & $\begin{array}{l}\text { Spikelet } \\
\text { fertility }\end{array}$ & $\begin{array}{l}\text { No. of filled } \\
\text { Grains } \\
\text { /panicle }\end{array}$ & $\begin{array}{c}1000 \\
\text { grain } \\
\text { weight }(\mathrm{g})\end{array}$ & $\begin{array}{c}\text { Yield } \\
\text { /plant } \\
\text { (g) }\end{array}$ & $\begin{array}{c}\text { Grain length } \\
\text {-breadth } \\
\text { ratio }\end{array}$ \\
\hline CMS-1×Benaful & 0.48 & 0.17 & 0.48 & $-1.38 * *$ & $14.42 * *$ & 0.01 & $11.60 * *$ & $0.628 * *$ & $-0.52 *$ & $-0.04 * *$ \\
\hline CMS- $1 \times$ Chinigura & -1.06 & $0.39 *$ & -0.35 & $-0.82 * *$ & -6.07 & -0.01 & $-5.28 *$ & $-0.909 * *$ & $0.58 *$ & $-0.04 * *$ \\
\hline CMS- $1 \times$ Kalijira-4 & -1.52 & $0.58 * *$ & -0.19 & $0.80^{*}$ & 4.75 & $0.02 * *$ & $6.89 * *$ & $-0.517 * *$ & $1.90 * *$ & $-0.10 * *$ \\
\hline CMS- $1 \times$ Kalijira- 7 & -2.82 & $-0.67 * *$ & $1.82 * *$ & $1.32 * *$ & $24.00 * *$ & $-0.01 *$ & $15.22 * *$ & -0.182 & 0.45 & $0.34 * *$ \\
\hline CMS-1×Kataribhog & 1.92 & 0.14 & $1.65 * *$ & $1.04 * *$ & $13.57 * *$ & $0.02 * *$ & $12.95 * *$ & $0.426^{* *}$ & $0.54 *$ & $-0.13 * *$ \\
\hline CMS- $1 \times$ Keora & 1.46 & $0.78 * *$ & $-1.19 *$ & 0.24 & $-24.50 * *$ & 0.01 & $-15.83 * *$ & $-0.357 * *$ & $-3.70 * *$ & $-0.31 * *$ \\
\hline CMS-1×Maloti-1 & -0.05 & $0.43 *$ & $0.98 *$ & $-1.53 * *$ & $-7.10^{*}$ & $-0.02 * *$ & -10.35 & $-1.211 * *$ & $0.59 *$ & $-0.09 * *$ \\
\hline CMS- $1 \times$ Oval Tapl & 1.81 & $-1.17 * *$ & -0.35 & 0.06 & $-17.63 * *$ & $-0.02 * *$ & $-15.79 * *$ & 0.024 & $-1.37 * *$ & $0.10 * *$ \\
\hline CMS- $1 \times$ Sorukamini- 1 & -0.22 & $-0.64 * *$ & $-2.85 * *$ & 0.28 & -1.43 & 0.01 & 0.64 & $2.098 * *$ & $1.53 * *$ & $0.27 * *$ \\
\hline CMS-2×Benaful & -0.48 & -0.18 & -0.48 & $1.38 * *$ & $-14.42 * *$ & -0.01 & $-11.57 * *$ & $-0.628 * *$ & $0.52 *$ & $0.04 * *$ \\
\hline CMS- $2 \times$ Chinigura & 1.06 & $-0.39 *$ & 0.35 & $0.82 * *$ & 6.07 & 0.01 & $5.28 *$ & $0.909 * *$ & $-0.58 *$ & $0.04 * *$ \\
\hline CMS-2×Kalijira-4 & 1.52 & $-0.58 * *$ & 0.19 & $-0.80 *$ & -4.75 & $-0.02 * *$ & $-6.89 * *$ & $0.517 * *$ & $-1.90 * *$ & $0.10 * *$ \\
\hline CMS-2×Kalijira-7 & 2.82 & $0.67 * *$ & $-1.82 * *$ & $-1.32 * *$ & $-24.00 * *$ & $0.01^{*}$ & $-15.22 * *$ & 0.182 & -0.45 & $-0.34 * *$ \\
\hline CMS-2×Kataribhog & -1.92 & -0.14 & $-1.65 * *$ & $-1.04 * *$ & $-13.57 * *$ & $-0.02 * *$ & $-12.95 * *$ & $-0.426 * *$ & $-0.54 *$ & $0.13 * *$ \\
\hline CMS- $2 \times$ Keora & -1.46 & $-0.78 * *$ & $1.19 *$ & -0.24 & 24.50 ** & -0.01 & $15.83 * *$ & $0.357 * *$ & $3.70 * *$ & $0.31 * *$ \\
\hline CMS-2×Maloti-1 & 0.05 & $-0.43^{*}$ & $-0.98 *$ & $1.53 * *$ & $7.10 *$ & $0.02 * *$ & $10.35^{* *}$ & $1.211^{* *}$ & $-0.59 *$ & $0.09 * *$ \\
\hline CMS-2×Oval Tapl & -1.81 & $1.17 * *$ & 0.35 & -0.06 & $17.63^{* *}$ & $0.02 * *$ & $15.80 * *$ & -0.024 & $1.37 * *$ & $-0.10 * *$ \\
\hline CMS- $2 \times$ Sorukamini- 1 & 0.22 & $0.64 * *$ & $2.85 * *$ & -0.28 & 1.43 & -0.01 & -0.64 & $-2.098 * *$ & $-1.53 * *$ & $-0.27 * *$ \\
\hline
\end{tabular}

$* *$ and $*$ significant at $1 \%$ and $5 \%$ level, respectively 
Table 4. Standard heterosis (\%) in aromatic hybrid rice for yield and yield contributing characters

\begin{tabular}{|c|c|c|c|c|c|c|c|c|c|c|}
\hline Hybrid & $\begin{array}{l}\text { Plant } \\
\text { height } \\
(\mathrm{cm})\end{array}$ & $\begin{array}{c}\text { No. of } \\
\text { productive } \\
\text { tillers /plant }\end{array}$ & $\begin{array}{l}\text { Days to } \\
\text { maturity }\end{array}$ & $\begin{array}{c}\text { Panicle } \\
\text { length } \\
\text { (cm) }\end{array}$ & $\begin{array}{l}\text { No. of total } \\
\text { spikelets } \\
\text { /panicle }\end{array}$ & $\begin{array}{l}\text { Spikelet } \\
\text { fertility }\end{array}$ & $\begin{array}{l}\text { No. of filled } \\
\text { grains } \\
\text { /panicle }\end{array}$ & $\begin{array}{l}1000 \text { grain } \\
\text { Weight (g) }\end{array}$ & $\begin{array}{c}\text { Yield / } \\
\text { plant (g) }\end{array}$ & $\begin{array}{c}\text { Grain length } \\
\text {-breadth } \\
\text { ratio }\end{array}$ \\
\hline CMS-1×Benaful & $4.47 *$ & $-5.62 * *$ & 0.69 & $6.05 * *$ & $-8.90 * *$ & 1.86 & $-7.37 * *$ & $38.65^{* *}$ & $4.22 * *$ & $10.28 * *$ \\
\hline CMS- $1 \times$ Chinigura & $4.90^{*}$ & $4.92 * *$ & $-3.90 * *$ & $14.83 * *$ & $-6.47 *$ & $-7.17 * *$ & $-13.44 * *$ & $13.42^{* *}$ & $5.06 * *$ & $-19.19 * *$ \\
\hline CMS- $1 \times$ Kalijira-4 & 1.14 & $-7.73 * *$ & $-2.2 * *$ & $17.89 * *$ & 4.65 & $3.58 * *$ & $8.32 * *$ & $13.90 * *$ & $12.54 * *$ & $-9.56 * *$ \\
\hline CMS- $1 \times$ Kalijiira-7 & -0.46 & $-8.20 * *$ & $-2.52 * *$ & $24.23 * *$ & $7.03 * *$ & $-10.60 * *$ & -4.22 & $23.60 * *$ & $4.32 * *$ & $15.82 * *$ \\
\hline CMS-1×Kataribhog & $4.30 *$ & $-4.68 *$ & -0.69 & $18.35 * *$ & $-7.10^{* *}$ & $3.58 * *$ & -4.24 & $33.80 * *$ & $-4.99 * *$ & $1.72 * *$ \\
\hline CMS- $1 \times$ Keora & $5.79 * *$ & $-6.09 * *$ & $-4.13 * *$ & $17.75^{* *}$ & $-32.27 * *$ & $-6.31 * *$ & $-36.54 * *$ & $44.72^{* *}$ & $-18.42 * *$ & $-5.67 * *$ \\
\hline CMS-1×Maloti-1 & $5.14 *$ & $-10.75 * *$ & $-1.83 * *$ & 2.93 & -3.27 & -1.15 & -4.65 & $16.10 * *$ & 0.47 & $-16.08 * *$ \\
\hline CMS-1×Oval Tapl & $4.89^{*}$ & $-20.14 * *$ & $-1.83 * *$ & $18.16^{* *}$ & $-15.93 * *$ & $-12.32 * *$ & $-26.12 * *$ & $53.31 * *$ & 1.16 & $6.49 * *$ \\
\hline CMS- $1 \times$ Sorukamini- 1 & $4.43 *$ & $-12.16 * *$ & $-4.59 * *$ & $10.89 * *$ & 4.04 & -0.72 & 3.36 & $44.38 * *$ & $5.12 * *$ & $7.56 * *$ \\
\hline CMS-2×Benaful & $5.11 *$ & $-4.92 * *$ & 0.69 & $13.50 * *$ & $-16.98 * *$ & 1.86 & $-15.63 * *$ & $8.88 * *$ & 1.12 & $4.93 * *$ \\
\hline CMS- $2 \times$ Chinigura & $7.95 * *$ & 2.58 & $-2.75 * *$ & $17.68 * *$ & $6.94 * *$ & $-5.45 * *$ & 1.11 & $3.00 *$ & $-4.83 * *$ & $-24.54 * *$ \\
\hline CMS-2×Kalijira-4 & $4.90 *$ & $-12.65 * *$ & $-1.38 * *$ & $7.40 * *$ & $6.72 *$ & -0.29 & $6.41 *$ & -1.45 & $-5.54 * *$ & $-11.41 * *$ \\
\hline CMS-2×Kalijira-7 & $5.36 * *$ & $4.45^{*}$ & $-4.36 * *$ & $9.44 * *$ & $-11.11 * *$ & $-6.74 * *$ & $-17.41 * *$ & 4.03 & $-4.78 * *$ & $-11.99 * *$ \\
\hline CMS-2×Kataribhog & 2.69 & -3.51 & $-2.29 * *$ & $5.88 * *$ & $-14.29 * *$ & 0.14 & $-14.37 * *$ & $6.57 * *$ & $-14.67 * *$ & $1.33 * *$ \\
\hline CMS-2×Keora & $4.90 *$ & $-13.82 * *$ & $-1.83 * *$ & $11.86 * *$ & 0.49 & $-8.03 * *$ & $-7.71 * *$ & $27.36^{* *}$ & -1.91 & $4.35 * *$ \\
\hline CMS-2×Maloti-1 & $6.61^{* *}$ & $-13.58 * *$ & $-2.52 * *$ & $11.61 * *$ & $11.23 * *$ & $4.87 * *$ & $16.76^{* *}$ & $9.49 * *$ & $-9.51 * *$ & $-18.51 * *$ \\
\hline CMS-2×Oval Tapl & 3.46 & -0.47 & -0.69 & $13.77 * *$ & $9.62 * *$ & $-6.31 * *$ & 2.66 & $31.14^{* *}$ & $3.31 * *$ & $-5.86 * *$ \\
\hline CMS- $2 \times$ Sorukamini- 1 & $6.17 * *$ & 0.00 & 0.00 & $4.66 * *$ & $12.50 * *$ & $-2.44 * *$ & $9.90 * *$ & $-3.91 * *$ & $-10.69 * *$ & $-16.08 * *$ \\
\hline Mean heterosis & 4.54 & -6.25 & -2.01 & 12.60 & -2.95 & $-2 . .87$ & -5.73 & 20.39 & -2.11 & -4.80 \\
\hline
\end{tabular}

** and $*$ significant at $1 \%$ and $5 \%$ level 
Most of the hybrids exhibited significant negative both types of heterosis for spikelet Highest value for standard heterosis were estimated as $44.38 \%$ (CMS- $1 \times$ Sorukamini- 1 ) and $53.31 \%$ (CMS-1 $\times$ Oval Tapl), respectively. Fifteen hybrids manifested desired significant standard heterosis for 1000 grain weight. The findings of Mishra and Pandey (1998) supported these results.

Eight cross combination expressed significant positive standard heterosis for grain length-breadth ratio. Seven hybrid exhibited significant standard heterosis for 1000-grain weight and grain length-breadth ratio. Of them, CMS- $1 \times$ Kalijira-7, CMS-1 $\times$ Oval Tapl and CMS- $1 \times$ Sorukamini- 1 were significant. Only CMS- $2 \times$ Keora exhibited desired significant standard heterosis for grain length-breadth ratio. While CMS-2 $\times$ Maloti-1 expressed significant positive standard heterosis for number of filled grains per panicle and 1000-grain weight. Yolanda and Das (1996) reported same on the extent of heterosis for number of filled grains per panicle and 1000-grain weight. Only CMS-1 $\times$ Kataribhog manifested desired significant pollen parent heterosis at a time for number of filled grains per panicle, 1000-grain weight and grain length-breadth ratio. None of the hybrids showed desired significant standard heterosis simultaneously for any three or more characters.

\section{REFERENCES}

Bansal, U. K., R. G. Saini and N. S. Rani. 2000. Heterosis and combining ability for yield, its components and quality traits in some scented rice (Oryza sativa). Trop. Agril. 77(3): 180-187.

Faiz, F. A., M. Sabar, T. H. Awan, M. Ijaz and Z. Manzoor. 2006. Heterosis and combining ability analysis in Basmati rice hybrids. J. Animal and Pl. Sci. 16(1/2): 56-59.

Fehr, W. R. 1987. Principles of cultivar development. Theory and echnique.Macmillan Pub. Co. Inc. New York. USA. pp.115-119.

Kempthrone, O. 1957. An introduction to genetic statistics. John Wiley and Sons, New York.

Mehla, I., S. S. Ajmer, D. V. S. Panwar and A. Singh. 2000. Combining ability studies for yield and its components in rice hybrids involving wild abortive (WA) source of CMS line. Agricult. Sci. Diquest. 20(3): 146-149.

Mishra, M. and M. P. Pandey. 1998. Heterosis breeding in rice for irrigated sub-humid tropic in north India. Oryza. 35(1): 8-14.

Panwar, D. V. S., R. Kumar, A. Singh and B. S. Mehla. 1998. Studding heterosis for grain and its components in hybrid rice. Intl. Rice Res. Notes. 23(2): 15.

Ramalingam, J., N. Nadarajan, C. Vanniarajan and P. Rangsamy. 1997. Combining ability studies involving CMS lines in rice. Oryza. 34: 4-7.

Ramesha, M. S., M. I. Ahmed, B. C. Viraktamath and C. H. M. Vijayakumar. 1999. Physiological basis of heterosis for grain yield in rice. Indian J. Genet. and Pt. Breed. 59(4): 411-415.

Sarker, U. K., M. G. Rasul and M. A. K. Mian. 2002. Heterosis and combining ability in rice. Bangladesh. J. Pl. Breed. Genet 15(1): 17-26.

Sitaramaiah, K. V., C. V. D. Rani and N. C. Reddi. 1998. Standard heterosis of rice hybrids for yield and yield components. Int. Rice Res. Newsl, 23(2): 15.

Surek, H. and K. Z. Korkut. 1996. Heterosis for yield and its components in rice (Oryza sativa L.) under temperate conditions. Bangladesh J. Pl. Breed. Genet. 9(1\&2): 3339.

Swamy, M. H., M. R. G. Rao and B. Vidyachandra. 2003. Studies on combining ability in rice hybrids involving new CMS lines. Karnataka J Agricult. Sci. 169 (2): 228-233.

Yadav, L. S., D. M. Maurya, S. P. Giri and S. B. Singh. 1999. Combining ability analysis for yield and its components in hybrid rice. Oryza. 36(3): 208-210.

Yolanda, J. L. and L. D. V. Das. 1996. Study on heterosis in hybrid rice. Oryza. 32: 109-110.

Yuan, L. P. 1998. Hybrid rice in China. 